\title{
An Updated Analysis of the Ocean Surface Wind Direction Signal in Passive Microwave Brightness Temperatures
}

\author{
Thomas Meissner, Member, IEEE, and Frank Wentz
}

\begin{abstract}
We analyze the wind direction signal for vertically $(v)$ and horizontally $(h)$ polarized microwave radiation at $37 \mathrm{GHz}$, $19 \mathrm{GHz}$, and $11 \mathrm{GHz}$ and an Earth incidence angle of $53^{\circ}$. We use brightness temperatures from SSM/I and TMI and wind vectors from buoys and the QUIKSCAT scatterometer. The wind vectors are space and time collocated with the radiometer measurements. Water vapor, cloud water and sea surface temperature are obtained from independent measurements and are uncorrelated with the wind direction. We find a wind direction signal that is noticeably smaller at low and moderate wind speeds than a previous analysis had indicated. We attribute the discrepancy to errors in the atmospheric parameters that were present in data set of the earlier study. We show that the polarization combination $2 v-h$ is almost insensitive to atmospheric changes and agrees with the earlier results. The strength of our new signals agrees well with recent JPL aircraft radiometer measurements. It is significantly smaller than the prediction of the two-scale sea surface emission model for low and intermediate wind speeds.
\end{abstract}

Index Terms-Microwave radiometers, ocean wind vector retrieval.

\section{INTRODUCTION}

$\mathbf{T}$ HE microwave radiation, which is emitted from the wind- roughened ocean surface and measured by satellite radiometer sensors, shows a small but distinct signature with respect to the wind direction relative to the radiometer azimuth. This is due to various anisotropic effects that the wind induces upon the sea surface. In general, the ocean surface contains three different types of roughness scales.

1) Large gravity waves, whose wavelengths are long compared with the radiation wavelength.

2) Small gravity-capillary waves, which are riding on top of the large-scale waves and whose RMS height is small compared with the radiation wavelength. These smallscale waves cause diffraction (Bragg scattering) of radiation that is backscattered from the ocean surface. From Kirchhoff's law it follows that they also affect the passive microwave emission of the sea surface.

3) Sea foam, which arises as a mixture of air and water at the wind roughened ocean surface and which leads to a general increase in the surface emissivity.

Manuscript received November 19, 2001; revised April 10, 2002. This work was supported by NASA Contract NAS5-32594 and by the Boeing/AER investigation for CMIS under IPO Contract F04-70197-C-0033.

The authors are with Remote Sensing Systems, Santa Rosa, CA 95401 USA (e-mail: meissner@remss.com; wentz@remss.com).

Publisher Item Identifier 10.1109/TGRS.2002.800231.
These different mechanisms exhibit several anisotropic features, which, in turn, lead to a wind directional dependence of the observed brightness temperatures. The probability density function of the sea surface slope is skewed in the alongwind axis and has a larger alongwind variance than crosswind variance [1]. Furthermore, the RMS height of the small gravity-capillary waves, which are riding on top of the large gravity waves, exhibits a noticeable anisotropy. The gravity-capillary waves traveling in the alongwind direction have larger amplitudes than those traveling in the crosswind direction [2]. Both effects cause an up-crosswind asymmetry of the emitted radiation. In addition, up-downwind asymmetries occur. The gravity-capillary waves and sea foam are not uniformly distributed over the underlying structure of large-scale waves. Aircraft radiometer measurements [3] show that the forward plunging side of a breaking wave is emitting warmer microwave emissions than its backside. Furthermore, the small-scale gravity-capillary waves have the tendency to cluster on the downwind side of the large-scale gravity waves [1], [4]. Finally, recent studies of nonlinear wave-wave interaction suggest that the small-scale ocean surface waves are not propagating in the wind direction [5]-[9].

An accurate knowledge of this directional dependence of the sea surface emissivity and the received brightness temperature is essential for several reasons. The wind direction signal constitutes an unwanted source of noise for the retrievals of wind speed and sea surface temperature (SST) from passive microwave radiometer sensors. This is important for the special sensor microwave/imager (SSM/I), the TRMM microwave imager (TMI) sensor of the tropical rainfall measurement mission (TRMM) and the advanced microwave scanning radiometers (AMSR) that started in May 2002. The more accurate the wind direction dependence of the brightness temperatures is known the better it is possible to eliminate the crosstalk between wind direction and the retrieved geophysical parameters. On the other hand, a large enough wind directional signature in the brightness temperatures opens the possibility of a radiometer measurement of the wind direction, similar to the ability of scatterometers to sense the wind direction using the distinct directional dependence of radar backscattering [10]-[16].

The first experimental analysis of the wind direction signal for vertical $(v)$ and horizontal $(h)$ polarized microwave radiation emitted by the ocean surface was done by Bespalova et al. [17] from nadir aircraft observation and by Wentz [18] for satellite observations at large incidence angles. Wentz [18] has used brightness temperatures that were measured by the SSM/I F08 sensor at $19.35 \mathrm{GHz}$ and $37.0 \mathrm{GHz}$ and an Earth incidence angle 
(EIA) of $\theta=53 \mathrm{deg}$. Over the period from July 1987 to March 1988 these brightness temperatures were collocated with wind vectors measured by 19 buoys from the National Data Buoy Center (NDBC). The spatial collocation was $50 \mathrm{~km}$ and the time collocation window $1.5 \mathrm{~h}$. Appropriate quality checks to exclude land or sea ice contamination, rain events or measurement errors were applied. The analysis contained about 3300 valid events. It found that for moderate wind speeds near $8 \mathrm{~m} / \mathrm{s}$ that the $v$-pol upwind is about $2 \mathrm{~K}$ higher than the $v$-pol downwind. There was no noticeable up-crosswind or down-crosswind asymmetry in the $v$-pol. For $h$-pol, the brightness temperature maximum occurred at crosswind and was approximately $3.5 \mathrm{~K}$ higher than the minimum at downwind and about $1.5 \mathrm{~K}$ higher than the upwind value.

The Jet Propulsion Laboratory (JPL) group has performed a series of measurements using the polarimetric 19 and $37 \mathrm{GHz}$ aircraft radiometer WINDRAD [19]-[23]. The most up to date version of their signal is given in [23], which extends the results that were published in [22] to higher wind speeds. In addition to measuring $v$ and $h$-pol radiance, it is also able to determine the third and fourth Stokes parameters and therefore the complete polarization state of the emitted radiation [24]. The most recent JPL results [22], [23] show almost no up-downwind asymmetry for the $h$-pol at low and moderate wind speeds in contrast to the SSM/I measurements. Moreover, the aircraft measurements show noticeably weaker $v$-pol up-downwind and $h$-pol downcrosswind signals at low and moderate wind speeds than the SSM/I measurements do.

Several theoretical attempts have been made to determine the wind direction signal from a two-scale ocean surface emission model, which incorporates the roughness effects 1 and 2 mentioned earlier. The ocean surface is approximated by a two-scale surface where small-scale gravity-capillary waves ride on top of large-scale gravity waves [25], [26]. If the curvature of the largescale waves is not too large, the geometric optics (GO) scattering model or Kirchhoff approximation can be used. Herein the large-scale waves are modeled as an ensemble of tilted facets each acting individually as an infinitely large specular reflecting surface. The RMS height of the small-scale gravity-capillary waves is treated as a perturbative parameter and the electric field of the EM wave at the ocean surface is expanded up to 2nd order [27]-[33]. A self contained quantitative study of the wind direction signal in all 4 Stokes parameters at 10.7, 19.35, and $37 \mathrm{GHz}$ has been performed by Poe and St. Germain [30]-[33]. Their results for the $v$-pol and the down-crosswind $h$-pol signals are close to the ones of [18]. On the other hand, similar as in [22], [23], their up-downwind $h$-pol signal is very small.

Since the original study of [18] a large number of additional data sets has become available. There are satellite measurements from 5 more SSM/I instruments (F10, F11, F13, F14, and F15) and, since 1998 also from TMI between 40S and $40 \mathrm{~N}$ latitude. The TMI contains two additional channels $(v$ and $h$-pol) at $10.7 \mathrm{GHz}$ and therefore allows the determination of the wind direction signal at lower frequencies. In addition, the sources for determining the wind vector have vastly increased. Data from the Tropical Atmosphere Ocean (TAO) network have become available and, since summer 1999, the NASA scatterometer QUIKSCAT provides very accurate ocean surface wind vector measurements over the whole globe. In view of the large uncertainties in shape and strength of the wind direction signal, it is therefore highly desirable to undertake an updated analysis using these new data sources and including the $11 \mathrm{GHz}$ TMI channels. This is the aim of this study.

Our paper is organized as follows:

Section II contains general aspects of the wind direction dependence of the sea surface emissivity and its attenuation by the Earth's atmosphere. In Section III we describe the general method of the analysis of the wind direction signal, show the possible problems associated with it and how to avoid these problems by using appropriate data sets. We also give a brief description of the data sets that we have used in our analysis. In Section IV we present our results and compare them with those of the previous studies. Section $\mathrm{V}$ discusses the influence of atmospheric parameters (water vapor and liquid cloud water) on the determination of the wind direction signal and points out some problems with the earlier analysis of [18]. Section VI concludes with a summary and a short discussion of the effects of our new results on wind vector retrieval algorithms in polarimetric radiometry.

\section{Wind Direction Dependence of Sea Surface EMISSIVITIES AND BRIGHTNESS TEMPERATURES}

\section{A. Harmonic Expansion of the Sea Surface Emissivity}

From reflection symmetry properties of Maxwell's equations [34] it follows that both $v$-pol and $h$-pol radiation emitted from the ocean surface are even and periodic functions of the relative wind direction $\varphi$, which is defined as the difference between geographic wind direction relative to North $\varphi_{w}$ and the azimuthal radiometer look angle $\alpha$. The surface emissivities for $v$ and $h$-pol can therefore be expanded into harmonic cosine (Fourier) series

$$
E_{i}=E_{i 0}+E_{i 1} \cdot \cos (\varphi)+E_{i 2} \cdot \cos (2 \varphi)+\cdots
$$

where $i=v, h$ denotes the polarization. The 0th harmonic coefficient $E_{i 0}$ is the isotropic (direction independent) part of the surface emissivity. It is a function of the Earth incidence angle (EIA), $\theta$, sea surface temperature (SST), $T_{s}$ salinity, $s$ and the surface wind speed, ${ }^{1}$ which is roughening the ocean surface. Our analysis excludes rain events so that we do not have to be concerned about the roughening of the sea surface by rain. The 1st harmonic coefficient $E_{i 1}$ characterizes the up-downwind asymmetry and the second harmonic coefficient $E_{i 2}$ the along-crosswind asymmetry in the surface emission. The $E_{i 1}$ and $E_{i 2}$ depend on the surface wind speed $W$ and the EIA $\theta$. It also has been found that higher order terms in the expansion (1) are small [18], [22], [31]. In the following, we will focus on the directional dependence:

$$
\Delta E_{i}=E_{i}-E_{i 0}
$$

\section{B. Atmospheric Attenuation and Radiative Transfer}

The brightness temperature $T_{B}$ that is received by the satellite radiometer consists of three parts.

\footnotetext{
${ }^{1}$ Throughout the paper the wind speed $W$ will be referenced to $10 \mathrm{~m}$ above the ocean surface.
} 
1) The signal that is emitted by the ocean surface and travels upward through the Earth's atmosphere.

2) The atmospheric radiation plus cold space radiation that travels downward and is scattered back from the ocean surface.

3) The upward traveling atmospheric radiation.

The microwave radiation is attenuated while traveling through the Earth's atmosphere. In the absence of rain, the atmospheric absorption and emission in the frequency range of interest are basically governed by three physical processes [34]-[43].

1) Oxygen absorption consisting of a set of rotational lines near $60 \mathrm{GHz}$ and an isolated line at $118.8 \mathrm{GHz}$. This process is characterized by the atmospheric profile for air pressure $p(z)$ and temperature $T(z)$. Here, the integration variable $z$ denotes the distance along some specified path through the atmosphere from the surface 0 to the satellite location $S$.

2) Water vapor absorption consisting of rotational lines at 22.2 GHz, $183.3 \mathrm{GHz}$, and several lines in the submillimeter wave region (above $300 \mathrm{GHz}$ ). This process can be characterized by the atmospheric water vapor density profile: $\rho_{v}(z)$.

3) Rayleigh absorption by cloud water droplets, whose radii are small compared with the radiation wavelength. This process can be characterized by the atmospheric liquid cloud water density profile: $\rho_{L}(z)$.

From the theory of radiative transfer [18], [44]-[46] one obtains for $T_{B}$

$$
T_{B}=T_{B U}+\tau E T_{s}+\tau R(1+\Omega)\left(T_{B D}+\tau T_{c}\right) .
$$

The $T_{B U}$ and $T_{B D}$ are the up- and downwelling atmospheric brightness temperatures and are given by the atmospheric integrals

$$
\begin{aligned}
& T_{B U}=\int_{0}^{\infty} d z \alpha(z) T(z) \tau(z, S) \\
& T_{B D}=\int_{0}^{\infty} d z \alpha(z) T(z) \tau(0, z) .
\end{aligned}
$$

Hereby $\alpha(z)$ is the total atmospheric absorption coefficient and $T(z)$ is the atmospheric temperature at location $z$. The atmospheric transmittance between $z_{1}$ and $z_{2}$ is given by

$$
\tau\left(z_{1}, z_{2}\right)=\exp \left(-\int_{z_{1}}^{z_{2}} d z \alpha(z)\right) .
$$

The second term in (3) represents the radiation that is emitted by the ocean surface and attenuated by the Earth's atmosphere. $\tau \equiv \tau(0, S)$ is the total atmospheric transmittance. The term $\tau R T_{B D}$ represents the downwelling atmospheric radiation that is reflected by the ocean surface and is transmitted back through the atmosphere to the satellite. The term $\tau T_{c}$ adds the small contribution due to the cold space temperature $T_{c}=2.7 \mathrm{~K}$, which is transmitted through the whole atmosphere before it is reflected at the ocean surface. The surface reflectivity $R$ is given by Kirchhoff's law

$$
R=1-E .
$$

The correction term proportional to $\Omega$ in (3) accounts for the increase of the reflected radiation due to surface scattering as compared with simple specular reflection. If there is no wind ( $W=0$ ) and the sea surface is smooth, then $\Omega=0$. At a wind speed of $7 \mathrm{~m} / \mathrm{s}$ typical values for $\Omega$ range from 0.04 at $7 \mathrm{GHz}$ $v$-pol to 0.05 at $37 \mathrm{GHz} v$-pol and from 0.07 at $7 \mathrm{GHz} h$-pol to 0.15 at $37 \mathrm{GHz} h$-pol. The maximum values occurring in the frequency range between 7 and $37 \mathrm{GHz}$ are about 0.14 for $v$-pol and 0.25 for $h$-pol.

The set of (3)-(5) represent a solution of the radiative transfer equation [36]

$$
\frac{\partial T_{B}}{\partial z}=-\alpha(z)\left[T_{B}(z)-T(z)\right]
$$

\section{Brightness Temperature Signal}

The wind direction dependence of the sea surface emissivities leads to a corresponding angular dependence of the received brightness temperatures with harmonic expansions

$$
\begin{aligned}
T_{B i}=t_{i 0}\left(\tau, W, T_{s}, s\right)+t_{i 1}( & \tau, W) \cdot \cos (\varphi) \\
& +t_{i 2}(\tau, W) \cdot \cos (2 \varphi)+\cdots
\end{aligned}
$$

and signals

$$
\Delta T_{B i}=T_{B i}-t_{i 0}
$$

analogous to (1) and (2), respectively.

In order to relate $\Delta T_{B i}$ (9) to $\Delta E_{i}$ in (2) we need to make some simplifications. If the temperature profile is equal to a constant, which characterizes an effective temperature for the ocean-atmosphere system

$$
T(z) \approx \mathrm{const} \approx T_{S} \equiv T_{e f f}
$$

the expressions in (4) simplify to

$$
T_{B U}=T_{B D}=(1-\tau) T_{e f f}
$$

and (3) becomes

$T_{B} \approx T_{e f f}-R \tau^{2} T_{e f f}+R \tau(1-\tau) \Omega T_{e f f}+(1+\Omega) R \tau^{2} T_{c}$.

The third term in (12) is small compared with the second term and can be neglected. The error in doing this is less than $4 \%$. The last term in (12) is very small and can be omitted as well. We can therefore write as a very good approximation

$$
T_{B} \approx\left(1-R \tau^{2}\right) T_{\text {eff }}
$$

From (13) and (6) we find for the relation between brightness temperature signal and surface emissivity signal

$$
\Delta T_{B} \approx \Delta E \tau^{2} T_{e f f}
$$

Though this approximate expression has been derived under various assumptions, it has been confirmed by a numerical Monte-Carlo simulation of rough surface scattering [47]. 


\section{Determination OF the Wind DiReCtion Signal From COLlocated MEASUREMENTS OF BRIGHTNESS TEMPERATURES AND WIND VECTORS}

\section{A. General Method}

The basic idea for determining the wind direction sea surface emissivity signal from radiometer brightness temperatures is rather simple. Satellite $T_{B}$ temperature measurements are space and time collocated with wind vectors $\left(W, \varphi_{W}\right)$ that are measured in situ by ocean buoys or, if available, by a scatterometer. Rain events and events that contain possible contamination from land or sea ice are filtered out. The events are stratified with respect to wind speed $W$ and relative wind direction $\varphi=\varphi_{W}-\alpha$. Harmonic functions of the form (8) are then fitted to the measured $T_{B} s$ through linear regressions. This determines the harmonic coefficients $t_{i h}, h=1,2, \ldots$ of the brightness temperature signals (8) and (9). Using (14) one finds the corresponding expansions (1) for the emissivity signal (2).

\section{B. Removal of Atmospheric Variations}

The problem inherent in this method is that atmospheric conditions as well as surface temperatures are varying with the wind direction leading to fluctuations in the dominant isotropic term (0th harmonic) $t_{i 0}$ of (8). Unfortunately, this term is very sensitive to changes in the atmospheric parameters. Increasing the columnar integrated atmospheric water vapor $V$ by $1 \mathrm{~mm}$ or the columnar integrated liquid cloud water $L$ by $0.1 \mathrm{~mm}$ will lead to an increase in the $37 \mathrm{GHz}$ isotropic brightness temperatures by about $0.5 \mathrm{~K}(1.0 \mathrm{~K})$ for $v$-pol $(h$-pol). Therefore, those atmospheric variations and the induced changes in the isotropic brightness temperature can easily swamp the whole wind direction signal, which is only in the order of a few Kelvin. It is therefore necessary to remove the isotropic part $t_{i 0}$ from (8) before doing the harmonic fit. In [18], [44]-[46] a model function has been derived for the isotropic part that is based on the theory of radiative transfer and empirical observations. Because the full atmospheric profiles for temperature $T$, water vapor density $\rho_{v}$ and liquid cloud water density $\rho_{L}$ are not readily available, a simplified version has been established that depends only on the effective temperature $T_{\text {eff }}$ of the ocean-atmosphere system (similar than in (10)) and the vertical columnar integrals for water vapor

$$
V=\int_{0}^{H} d h \rho_{v}(h)
$$

and for liquid cloud water

$$
L=\int_{0}^{H} d h \rho_{L}(h)
$$

Here, $h$ denotes the vertical distance from sea level and $H$ is the satellite altitude. In order to obtain expressions for the specular sea surface emissivity we use empirical derived expressions for the complex dielectric constant of sea water, which depend on SST. The salinity $s$ is set to $s=35$ ppt. The GO (Kirchhoff) approximation is used for modeling large gravity waves. Diffraction effects by small gravity-capillary waves and sea foam are again treated empirically. For fixed EIA, the model function $F_{i}$ for polarization $i$ depends therefore on $T_{e f f}, V, L$, and $W$. The signal (9) is then determined as

$$
\Delta T_{B i}=T_{B i}-F_{i}\left(T_{e f f}, V, L, W\right) .
$$

For our new analysis, we have used the most updated version of the isotropic model function, which can be found in [46]. This model function is able to reproduce the SSM/I brightness temperatures below $37 \mathrm{GHz}$ to a RMS accuracy of $0.6 \mathrm{~K}$ [45].

\section{Appearance and Removal of Spurious Directional Signals}

Neither the functional form for $F_{i}$ nor the values for $T_{e f f}, V$, $L$ and $W$ are exact. This will introduce an error in (17). As long as this error is purely random Gaussian noise and not in any way correlated with the wind direction $\varphi$, the harmonic fit for $\Delta T_{B i}$ is not affected, but only the scatter in the $\Delta T_{B i}$ is increased. On the other hand, any correlation between the error $\delta F_{i}$ and the wind direction $\varphi$ will result in a spurious wind direction dependence of $\Delta T_{B i}$. As a consequence, it is essential to use values for $T_{e f f}, V, L$, and $W$ in (17) that are independent of $\varphi$. As it was pointed out in [18], this poses a problem for $V$ and $L$, if one obtains them from the same instrument that measures the brightness temperatures. The values for $V$ and $L$ have a small error due to neglecting the wind direction signal $\Delta T_{B i}$ in the retrieval algorithm. This error is naturally correlated with $\varphi$. The preferred method is to obtain them from a third independent data source, which is space and time collocated with the already existing data set for $T_{B}$ and $\left(W, \varphi_{W}\right)$. What comes into mind is to use another satellite, whose azimuthal look angle is uncorrelated with the azimuthal look angle of the satellite that measures the $T_{B}$. In the absence of available independent measurements for the atmospheric parameters, [18] proposed a retrieval method for $V$ and $L$ that uses only the brightness temperature differences $19 v-22 v, 37 v-22 v$, and $37 h-19 h$. Under the assumption that the wind direction signal is constant above $19 \mathrm{GHz}$ the wind direction effect will cancel out in these differences rendering retrieval results for the atmospheric parameters. These results are less accurate than the ones obtained from absolute brightness temperatures but uncorrelated with wind direction. Unfortunately, as we will discuss in Section V, there are still problems with this method in certain cases, which can cause the appearance of spurious wind direction effects in $\Delta T_{B i}$.

\section{D. $2 v-h$}

It will be instructive for our analysis to consider the polarization combination $2 v-h$ of the brightness temperature and emissivity signals. This special combination is largely independent of variations in the atmospheric parameters $V$ and $L$. It is therefore much less prone to the appearance of spurious atmospheric contaminations of the wind direction signal. To see this, we consider the change of the total received brightness temperature (13) under a change of the atmospheric parameters

$$
\delta T_{B}=-2 \tau R T_{\text {eff }} \delta \tau .
$$

This means that the brightness temperature error due to atmospheric fluctuations is directly proportional to the surface reflectivity $R$. The change in the atmospheric transmittance is caused by changing water vapor or liquid cloud water. At an EIA of $53^{\circ}$ the reflectivity of the ocean surface is about twice as large 
TABLE I

Collocated Data Sets Consisting of Brightness Temperatures $T_{B}$, Wind Speed $W$, Relative Wind Direction $\varphi$, Columnar Water Vapor $V$, Columnar Liquid Cloud Water $L$, and Sea Surface Temperature (SST)

\begin{tabular}{l|l|l|l}
\hline & Set 1 & Set 2 & Set 3 \\
\hline$T_{B}$ & SSM/I & TMI & SSM/I \\
& F13 & & F11+F13+F14 \\
& 0.25 deg maps & 0.25 deg maps & 0.25 deg maps \\
\hline$W, \varphi$ & OUIKSCAT & OUIKSCAT & BUOYS (TAO+NDBC) \\
& 0.25 deg maps & 0.25 deg maps & \\
\hline$V, L$ & TMI & SSM/I F13 & TMI \\
& 0.25 deg maps & 0.25 deg maps & 0.25 deg maps \\
\hline SST & TMI & REYNOLDS & TMI \\
& 0.25 deg maps & weekly maps & 0.25 deg maps \\
\hline Time Window & SSM/I - QUIKSCAT: 30 min & SSM/I - QUIKSCAT: 30 min & SSM/I - BUOY: I20 min \\
& SSM/I - TMI: 30 min & SSM/I - TMI: 30 min & linear interpolation between \\
& TMI - QUIKSCAT: 30 min & TMI - QUIKSCAT: 30 min & SSM/I and BUOY \\
& & & SSM/I - TMI: 120 min \\
\hline Time period of analysis & AUG1999-MAR2000 & AUG1999-MAY2000 & JAN1998 - MAR2000 \\
\hline \# of valid collocations events & $\approx 830,000$ & $\approx 1,030,000$ & $\approx 7,600$ \\
\hline
\end{tabular}

TABLE II

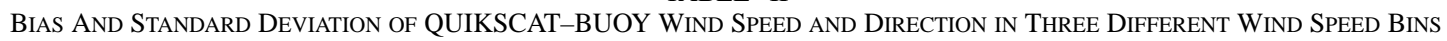

\begin{tabular}{l|l|l|l|l}
\hline Wind Speed Bin [m/s] & \multicolumn{2}{|c|}{ Wind Speed [m/s] } & \multicolumn{2}{c}{ Wind Direction [deg] } \\
\cline { 2 - 5 } & BIAS & SDEV & BIAS & SDEV \\
\hline$[0,6]$ & +0.18 & 0.79 & +4.7 & 35.9 \\
\hline$[6,10]$ & -0.11 & 0.67 & +6.9 & 11.0 \\
\hline$[10,14]$ & -0.17 & 0.72 & +5.6 & 10.5 \\
\hline
\end{tabular}

for $h$-pol than for $v$-pol and therefore any atmospheric variation will approximately cancel out in the combination $2 v-h$.

\section{E. Collocated Data Sets and Quality Checks}

The three collocated data sets that we have used in our analysis are summarized in Table I.

1) Set 1 uses brightness temperatures from SSM/I F13, wind vectors from QUIKSCAT and water vapor, cloud water and SST from TMI that were collected over the time period AUG 1999-MAR 2000. The geophysical parameters have been retrieved by Remote Sensing System's algorithms [45] and are available for the public on our website http://www.remss.com. The data from all three instruments have been processed into $0.25^{\circ}$ lat-lon maps allowing an easy spatial collocation. For time collocation, we require that the time difference between any two measurements is less than $30 \mathrm{~min}$. As indicated above, we consider only rain free atmospheres. Rain can produce large variations in the atmospheric brightness temperatures. That makes it difficult to extract the wind direction signal. It also would require an accurate knowledge of the rain rate, which is not easy to obtain [48]. Moreover, heavy rain roughens the sea surface itself and thereby changes its emissivity. An event is flagged as possible rain if the liquid cloud water density $L$ exceeds $0.18 \mathrm{~mm}$. In order to avoid any contamination from either rain as well as land or sea ice pixels, we neglect any cell if there is rain, land or ice present in any one of its eight adjacent cells. Essential for the quality of the analysis is the quality of the QUIKSCAT wind vectors, especially the wind di- rection. The differences between QUIKSCAT and in situ (buoy) measurements are summarized in Table II. In order to avoid outliers, we also discard any event for which any two of the SSM/I, QUIKSCAT and TMI retrieved wind speeds differ by more than $3.0 \mathrm{~m} / \mathrm{s}$. We have checked that including these outliers would not change our results for the wind direction signal but only increase the scatter in the data. The quality of the radiometer retrieved atmospheric parameters is about $1 \mathrm{~mm}$ for $V$ and about $0.025 \mathrm{~mm}$ for $L$ [45]. The whole data set 1 comprises about 830000 valid events. As we have discussed in the last section, it is important that the azimuthal look angles of the radiometers that measure the brightness temperatures and the atmospheric parameters are uncorrelated in order to avoid the appearance of spurious wind direction effects. SSM/I is a polar orbiting instrument, whereas TMI is an equatorial orbiting instrument, whose observations are restricted between $40 \mathrm{~S}$ and $40 \mathrm{~N}$ latitude. We have checked that the difference in their azimuthal look angles has indeed the distribution of a uniform random variable. We want to note that this would not be the case, if we had obtained $V$ and $L$ from a different SSM/I instrument, which can be reasonably time collocated with SSM/I F13, rather than from TMI. For example, the average azimuth angle difference between F13 with a local equatorial crossing time of 5:52 and F11 with a local equatorial crossing time of 7:24 has a standard deviation of only about $25^{\circ}$.

2) In set 2 , the radiometers measuring brightness temperatures and the atmospheric parameters have been interchanged from set 1 , i.e., we use TMI brightness temper- 
atures, QUIKSCAT wind vectors and SSM/I F13 water vapor and cloud water. In this case, the SST is taken from the weekly Reynolds maps [49]. As in the case of the atmospheric parameters, we need to use a product from a source that is uncorrelated with the radiometer, which is measuring the brightness temperatures. Otherwise, the collocation procedure is the same as for set 1 . The analysis time period is slightly larger ranging from AUG 1999 to MAY 2000, which renders about 1.030000 valid events. An advantage of using this data set is that it allows a determination of the wind direction signal at $11 \mathrm{GHz}$ together with the signals at the higher frequencies.

3) Set 3 uses SSM/I brightness temperatures in connection with wind vector measurements by buoys from the NDBC and TAO networks and TMI water vapor, cloud water and SST. Observations from 3 satellites F11, F13 and F14 over the time period from JAN 1998 until MAR 2000 have been included. For space collocation, we require that the reported geographic coordinates of the buoy lie within the $0.25^{\circ}$ latitude-longitude SSM/I and TMI pixel. The buoy events are generally recorded at every hour or every second hour. For time collocating SSM/I and buoy events we require that the two buoy events, which occur before and after the SSM/I event, are no more than 120 min apart. We perform a linear time interpolation of these 2 buoy wind vectors to the SSM/I time. The time cutoff between $\mathrm{SSM} / \mathrm{I}$ and TMI measurements is set to $120 \mathrm{~min}$. The events are filtered for rain, land and sea ice as it was done with sets 1 and 2 . In order to avoid outliers, we also neglect events for which the difference between the time-interpolated buoy wind speed and the TMI retrieved wind speed is larger than $3.0 \mathrm{~m} / \mathrm{s}$. Set 3 contains about 7,600 valid events.

\section{RESULTS}

\section{A. Shape and Size of the $37 \mathrm{GHz}$. Signal at Low, Intermediate and High Wind Speeds}

In order to examine form and shape of the signal we first bin the collocated events in each data set according to wind speed into the three intervals: Between 0 and $6 \mathrm{~m} / \mathrm{s}$, between 6 and $10 \mathrm{~m} / \mathrm{s}$ and between 10 and $14 \mathrm{~m} / \mathrm{s}$. Within each of these wind speed bin the events are then stratified with respect to the relative wind direction using 18 directional bins of $20^{\circ}$ size each. Then in each bin the average emissivity signal (2) is computed for both v-pol and h-pol and a harmonic form (1) is fitted by linear regression. Fig. 1 shows the result for the $37 \mathrm{GHz}$ channels in comparison with the ones of [18]. Throughout the paper, we will present emissivity signals that have been multiplied by a standard value effective temperature of $T_{\text {eff }}=293 \mathrm{~K}$. Due to (14), this corresponds to a brightness temperature signal (9) at this effective temperature for a completely transparent atmosphere $\tau=1$.

We see that the results of the three data sets agree very well with each other. The scatter within data set 3 is naturally much larger than within set 1 or set 2 , because set 3 contains many fewer events. This renders a correspondingly larger noise, but as
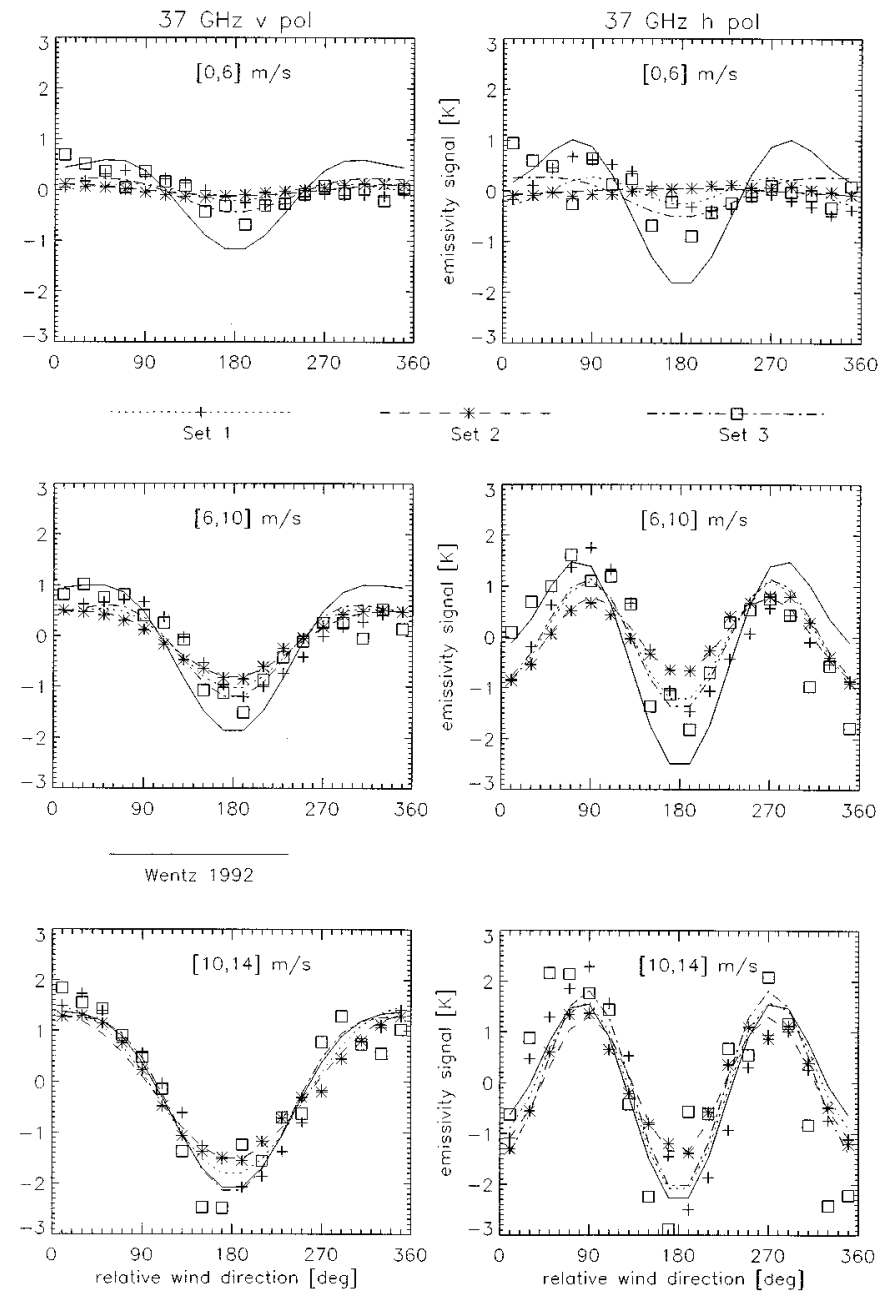

Fig. 1. Wind direction signal of the sea surface emissivity $\Delta E$ $T_{e f f}\left(T_{e f f}=293 \mathrm{~K}\right)$ for $37 \mathrm{GHz} v$ and $h$-pol in three different wind speed bins. The figure shows separately the results (data points) for the three data sets of Table I together with their harmonic fits (lines). The solid line is the harmonic fit of [18].

long as this noise is purely random (Gaussian), the final results for the harmonic fits are not affected.

There are, however, substantial differences to the former analysis [18]. None of the three data sets shows a significant signal at the lowest wind speeds. In the 2nd wind speed bin, the $v$-pol up-downwind and the $h$-pol along-downwind asymmetries are only about half as large as the ones in [18]. Moreover, we do not find any noticeable up-downwind asymmetry for the $h$-pol in this wind speed bin. In the third wind speed bin there is very good agreement between [18] and our new analysis.

\section{B. Comparison of the Harmonic Coefficients for $v, h$ and $2 v-h$ at $37 \mathrm{GHz}$}

For a more detailed account of the wind speed dependence of the wind direction signal, we have refined the wind speed stratification using six bins between 0 and $14 \mathrm{~m} / \mathrm{s}$. The first bin contains wind speeds between 0 and $4 \mathrm{~m} / \mathrm{s}$ and all the other intervals are $2 \mathrm{~m} / \mathrm{s}$ wide. In case of set 3 , the two highest wind speed bins are sparsely populated, containing only about 470 events between 10 and $12 \mathrm{~m} / \mathrm{s}$ and about 80 events between 12 and $14 \mathrm{~m} / \mathrm{s}$. This leads again to a relative large data scatter in 

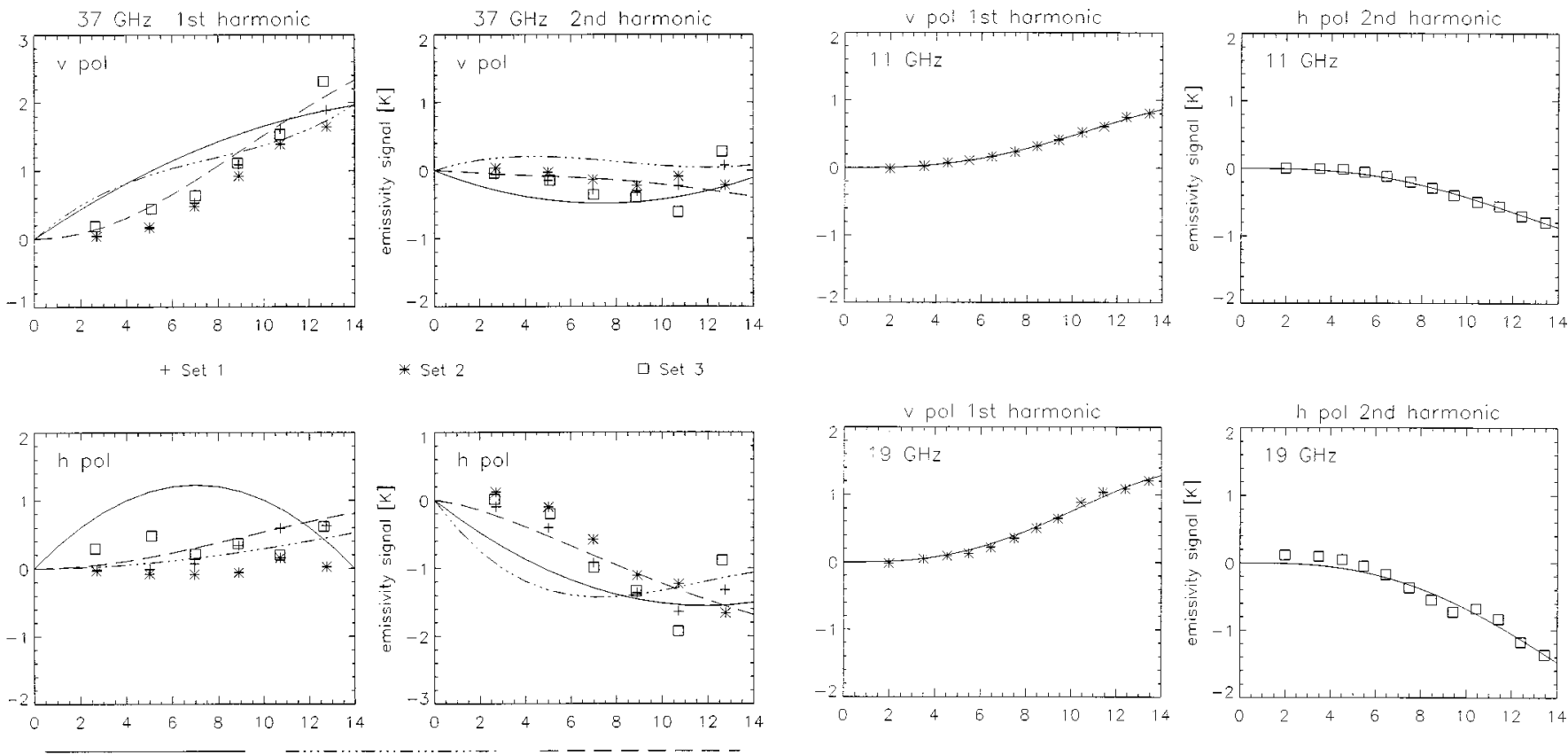

* Set 2
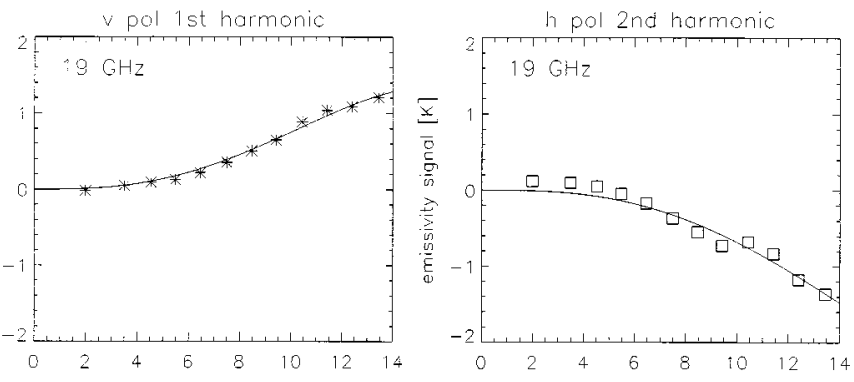

Wentz 1992

St. Germain + Poe 1998 Yueh + Wilson 1999
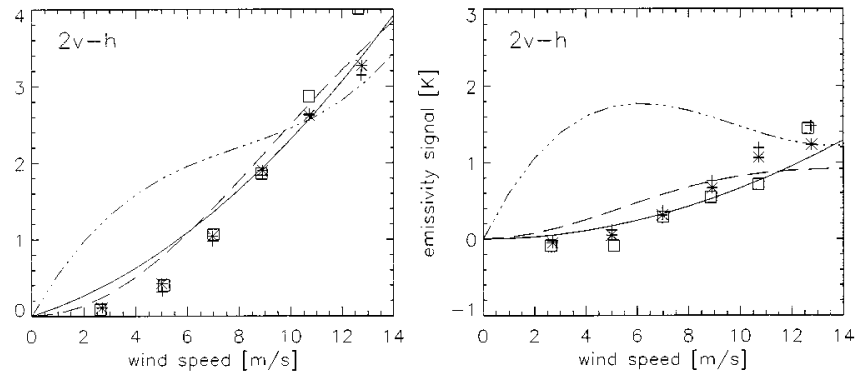

Fig. 2. Dependence of the wind direction signal $\Delta E \cdot T_{\text {ef }}\left(T_{e f f}=293 \mathrm{~K}\right)$ with wind speed. The figure shows the first and second harmonic coefficients at $37 \mathrm{GHz} v$-pol, $h$-pol and the combination $2 v-h$ for the three data sets of Table I (data points). Also shown are the results of [18], [31], and [23] (lines).

those cases. All the lower wind speed bins in set 3 contain more than 1200 events. The wind direction stratification in each wind speed bin is the same as in section A. For a fixed wind speed bin, we compute again the average emissivity signals $\Delta E_{i}$ in each directional bin and perform a harmonic fit. This renders values for the harmonic coefficients $E_{i 1}$ and $E_{i 2}$ in (1) as functions of wind speed. Fig. 2 shows the results at $37 \mathrm{GHz}$ for $v$ and $h$-pol as well as for the combination $2 v-h$. In addition, we have also plotted the values that were obtained by [18], by the recent JPL aircraft radiometer (WINDRAD) measurements [22], [23] and by the two-scale surface emission model [31]. As one can see, all of our three data sets agree again well with each other and are also very close the JPL aircraft measurements. If compared with [18] we find again substantially smaller dominant harmonics $v$ and $h$-pol at low and intermediate wind speeds, basically no 1 st harmonic $h$-pol, but generally good agreement at higher wind speeds. Moreover, the results for the combination $2 v-h$ are agree very well between [18] and our three data sets over the whole wind speed range. We will come back to this point in Section V. We also note that the two-scale scattering model [31] gets a different result for the combination $2 v-h$.
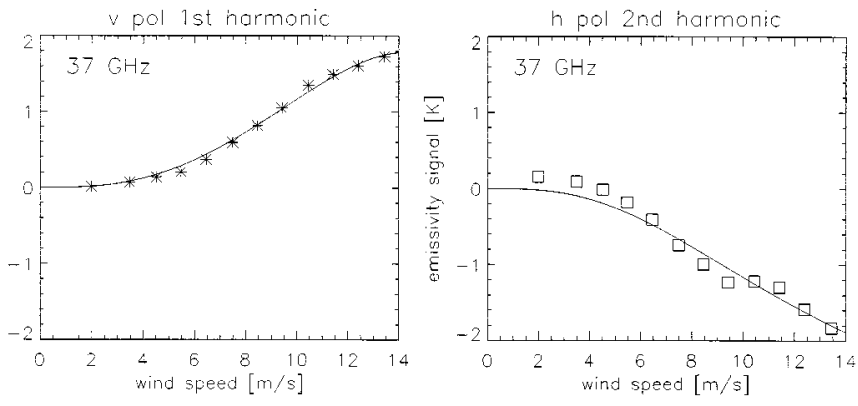

Fig. 3. Dependence of the wind direction signal $\Delta E \cdot T_{\text {eff }}\left(T_{e f f}=293 \mathrm{~K}\right)$ with wind speed at three different frequencies $(11 \mathrm{GHz}, 19 \mathrm{GHz}$, and $37 \mathrm{GHz})$ for the data set 2 of Table I. The figure shows the results for the dominant harmonics ( $v 1$ and $h 2$ ) (data points) together with fits of the functional forms (19) (lines). The nondominant harmonics ( $v 2$ and $h 1$ ) are compatible with zero in the wind speed interval between $0 \mathrm{~m} / \mathrm{s}$ and $14 \mathrm{~m} / \mathrm{s}$.

\section{Frequency Dependence}

We have repeated the analysis for the $11 \mathrm{GHz}$ and $19 \mathrm{GHz}$ channels using set 2 , because only the TMI brightness temperatures allow a determination of the signal at $11 \mathrm{GHz}$. We found that the signal between $11 \mathrm{GHz}$ and $37 \mathrm{GHz}$ increases nearly linearly with the logarithm of the frequency and the increase is almost identical for $v$ and $h$-pol. The amplitudes of the emissivity signals at $19 \mathrm{GHz}$ and $11 \mathrm{GHz}$ are about $70 \%$ and $40 \%$, respectively, of the amplitude at $37 \mathrm{GHz}$.

\section{Functional Fits of the Harmonic Coefficients at 11, 19, and $37 \mathrm{GHz}$}

Fig. 3 displays the dominant harmonic emissivity coefficients for $v$ and $h$-pol at 11, 19 and $37 \mathrm{GHz}$. The data were obtained from set 2 using 11 wind speed bins between 3 and $14 \mathrm{~m} / \mathrm{s}$ plus one bin for very low winds between 0 and $3 \mathrm{~m} / \mathrm{s}$. It turns out that the data can be well fitted with a functional form

$$
E_{i h}^{\nu}(W)=a_{i h}{ }^{\nu}\left[\exp \left(-\alpha_{i h}{ }^{\nu} W^{2}\right)-1\right]\left(b_{i h}{ }^{\nu} W+c_{i h}{ }^{\nu} W^{2}\right)
$$


TABLE III

COEFFICIENTS OF THE DOMINANT HARMONICS OF THE FUNCTIONAL FORM (19) FOR THREE FREQUENCIES (11 GHz, $19 \mathrm{GHz}$ AND $37 \mathrm{GHz}$ ) AT Two POLARIZATIONS ( $v$-POL AND $h$-POL). SET 2 OF TABLE II HAS BEEN USED

\begin{tabular}{l|l|l|l|l|l}
\hline Frequency & POL/HARM & a & $\alpha$ & b & c \\
\hline \multirow{3}{*}{$11 \mathrm{GHz}$} & V1 & $2.93 \mathrm{E}+00$ & $2.78 \mathrm{E}-05$ & $-1.06 \mathrm{E}+01$ & $4.81 \mathrm{E}-01$ \\
\cline { 2 - 6 } & $\mathrm{H} 2$ & $-2.30 \mathrm{E}+00$ & $2.97 \mathrm{E}-05$ & $-1.04 \mathrm{E}+01$ & $4.09 \mathrm{E}-01$ \\
\hline \multirow{3}{*}{$19 \mathrm{GHz}$} & V1 & $9.25 \mathrm{E}+00$ & $1.11 \mathrm{E}-05$ & $-1.42 \mathrm{E}+01$ & $6.85 \mathrm{E}-01$ \\
\cline { 2 - 6 } & $\mathrm{H} 2$ & $-2.37 \mathrm{E}-01$ & $6.60 \mathrm{E}-04$ & $-6.63 \mathrm{E}+00$ & $2.11 \mathrm{E}-01$ \\
\hline \multirow{2}{*}{$37 \mathrm{GHz}$} & $\mathrm{V} 1$ & $1.23 \mathrm{E}+01$ & $1.21 \mathrm{E}-05$ & $-1.64 \mathrm{E}+01$ & $8.57 \mathrm{E}-01$ \\
\cline { 2 - 6 } & H2 & $-1.58 \mathrm{E}-02$ & $1.75 \mathrm{E}-02$ & $-9.24 \mathrm{E}+00$ & $2.94 \mathrm{E}-02$ \\
\hline
\end{tabular}

where $\nu=11,19,37 \mathrm{GHz}$ indexes the frequency $i=v, h$ the polarization and $h=1,2$ the harmonic order. Equation (19) is valid for wind speeds between $0 \mathrm{~m} / \mathrm{s}$ and $14 \mathrm{~m} / \mathrm{s}$. The nondominant harmonics ( $v 2$ and $h 1)$ are compatible with zero in this interval. The values for the coefficients $a, \alpha, b, c$ are listed in Table III.

\section{The Wind DiRection Signal AND ATMOSPHERIC VARIATIONS}

Finally, we explain the discrepancies between the analysis of [18] and our new analysis. As we have seen in Fig. 2, the results for the combination $2 v-h$ coincide in both analyzes and moreover coincide also with the JPL WINDRAD [22], [23] measurements. However, the $v$-pol and $h$-pol themselves show significant differences, as we have mentioned in the last section. We have shown in Section III-D, that the combination $2 v-h$ is approximately independent of the atmospheric parameters $V$ and $L$ for an EIA of $53^{\circ}$. This suggests that the discrepancies between [18] and our new analysis are caused by errors in $V$ and $L$ in the data set of [18]. These errors remain somehow correlated with wind direction despite the attempt to remove them by retrieving $V$ and $L$ from brightness temperature differences (cf. Section III-C). In order to prove this assumption we have repeated the analysis of [18] and collocated SSM/I F08 $T_{B}$ with wind vectors from the 19 NDBC buoys. We have extended the period to the whole lifetime of F08 (JUL1987-DEC 1991) resulting in about 7900 collocated events. The atmospheric parameters are retrieved in the same way as in [18] using its version of the isotropic model function $F$ and the brightness temperature difference method. In Fig. 4 we have plotted the values for $V$ and $L$ in each of the $20^{\circ}$ wind direction intervals for wind speeds between 6 and $10 \mathrm{~m} / \mathrm{s}$ comparing our three sets from Table I with the F08-NDBC set. One recognizes strong up-downwind and along-downwind differences for both $V$ and $L$ within the F08-NDBC set.

The $T_{B}$ difference method for retrieving $V$ and $L$ is based on the assumption that the $T_{B}$ directional signal is constant above $19 \mathrm{GHz}$ and therefore any wind direction dependence cancels out after taking the differences between the 19,22 , and $37 \mathrm{GHz}$ channels. As we have seen in Section IV-C, this is not the case, but the $19 \mathrm{GHz} T_{B}$ signal is about $20 \%$ smaller than the $37 \mathrm{GHz}$ signal. In order to avoid any possible crosstalk between wind direction and the retrieved atmospheric parameters we have repeated the analysis after subtracting the wind direction signal from the measured $T_{B}$ in the F08-NDBC data set. Hereby we have used the results of our new analysis from Section IV-D [(19) and Table III] in connection with (14), (2), and (1). In
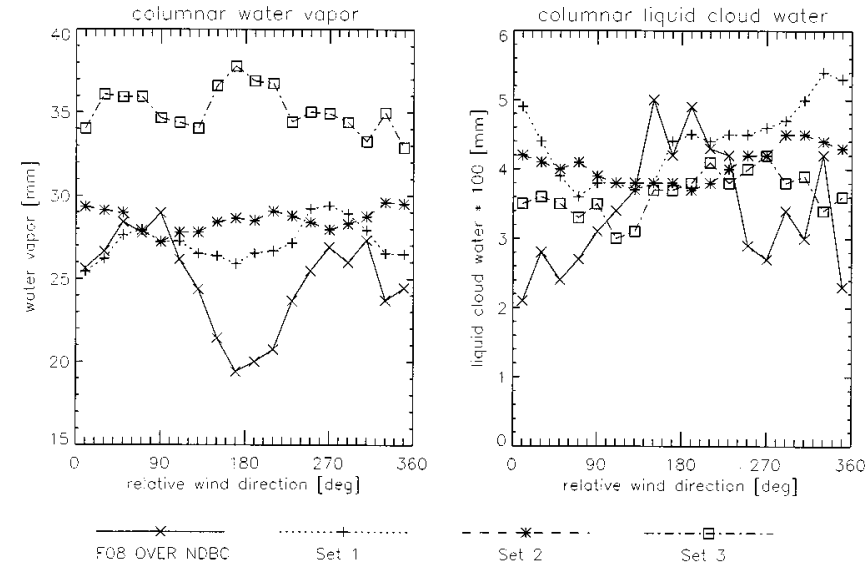

Fig. 4. Water vapor and columnar liquid cloud water in the wind speed bin between 6 and $10 \mathrm{~m} / \mathrm{s}$ as a function of relative wind direction. The figure shows the results for the three data sets of Table I and the data set of [18].

order to compute a brightness temperature signal we have set the atmospheric transmittance $\tau$ to a typical value of 0.9 [18], [22]. This is sufficiently accurate for our purposes, because a small error in the atmospheric part of the already small directional signal will not influence the results for $V$ and $L$. After removing the wind direction dependence we retrieve $V$ and $L$ for the F08-NDBC set using (1) $T_{B}$ difference method (labeled as case 1) and (2) absolute $T_{B}$ for $22 \mathrm{v}$ and $37 \mathrm{v}$ together with the buoy wind speed (labeled as case 2). Details of this retrieval algorithm can be found in Section IV and Section V of [18]. The retrievals of case 2 should be more accurate than the ones of case 1 , because case 2 is based on absolute $T_{B}$ values rather than on the small $T_{B}$ differences. Assuming that the result of our new analysis from Section IV-D describes the wind direction dependence correctly, neither case 1 nor case 2 should have any wind direction crosstalk in the retrieved values for $V$ and $L$.

Fig. 5 displays again $V$ and $L$ for both cases in the 2nd wind speed bin and in addition the $v$-pol and $h$-pol emissivity signals. First, it shows that the variation of $V$ with $\varphi$ is indeed a genuine feature of the F08-NDBC data set and not an artefact of the retrieval method, because it is obtained in both cases. The relative small number of events in this data set together with the geolocation of the buoys and looking geometry of F08 leads to the observed $V(\varphi)$ dependence. However, the values for $L$ differ strongly in both cases. This is causing the different result for the emissivity signals. In fact, case 1 gives basically the same results for the emissivity signals as [18], whereas case 2 gives basically the same result as our new analysis.

The liquid cloud water error, i.e., the difference of $L$ between case 1 and case 2 has a strong dependence on the value of $V$. The correlation between $V$ and $\varphi$ in the F08-DBC data set (Fig. 5) therefore results in a corresponding correlation between $\Delta L$ and $\varphi$. This, in turn, leads to an error in the isotropic model function $\Delta F(\varphi)$ and, as we have seen in Section III-C to a spurious, unwanted dependence $\Delta T_{B}(\varphi)$. In Fig. 6 we have plotted the differences $\Delta L, \Delta V$ and $\Delta F_{37}$ between case 1 and case 2 . We see that the model function difference $\Delta F$ corresponds indeed to the observed differences in the $T_{B}$ wind direction signal.

We must conclude that the $T_{B}$ difference method for retrieving the atmospheric parameters, if applied to a small data 

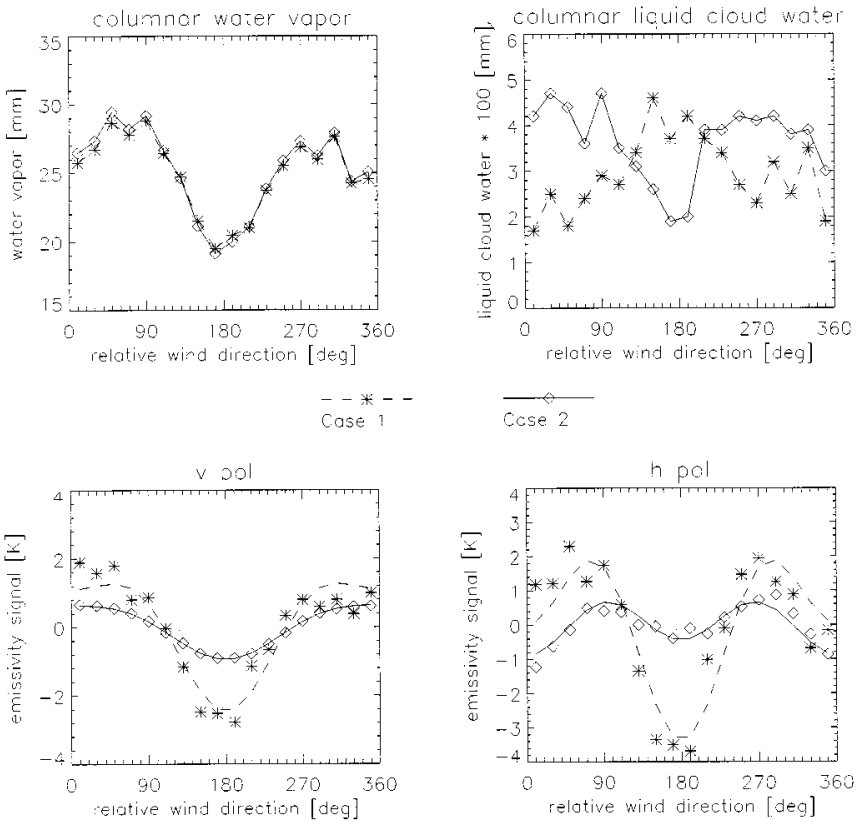

Fig. 5. Columnar water vapor, columnar liquid cloud water, $v$-pol, and $h$-pol emissivity signals at $37 \mathrm{GHz}$ in the wind speed bin between 6 and $10 \mathrm{~m} / \mathrm{s}$ as a function of relative wind direction for the data set of [18] (SSM/I over 19 NDBC buoys). Case 1 uses brightness temperature differences $37 \mathrm{v}-22 \mathrm{v}, 37 \mathrm{~h}-19 \mathrm{~h}$, and $22 \mathrm{v}-19 \mathrm{v}$ for retrieving water vapor and liquid cloud water. In case 2, water vapor and liquid cloud water are retrieved using the absolute $22 \mathrm{v}$ and $37 \mathrm{v}$ brightness temperatures and the buoy wind speeds. In both cases, the wind direction signal has been removed from the brightness temperatures.

$$
\text { Case i - Cose } 2
$$
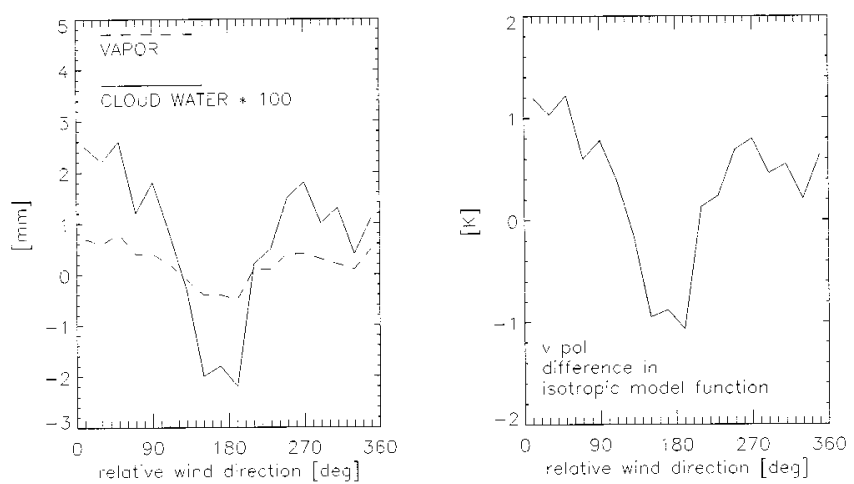

Fig. 6. Differences between case 1 and case 2: Water vapor, liquid cloud water and the $37 \mathrm{GHz}$ isotropic $v$-pol model function in the wind speed bin between $6 \mathrm{~m} / \mathrm{s}$ and $10 \mathrm{~m} / \mathrm{s}$ for the F08-NDBC data set as a function of relative wind direction.

set such as the F08-NDBC collocations, whose water vapor and liquid cloud water varies strongly with wind direction, can lead to a systematic error in the order of $+/-1 \mathrm{~K}$. This underlines the importance to obtain values for $V$ and $L$ from independent measurements, which are uncorrelated with the $T_{B}$ measurements.

\section{SUMMARY AND CONCLUSIONS}

We have studied the dependence of the ocean surface microwave emissivity at 11, 19, and $37 \mathrm{GHz}$ for vertical and horizontal polarizations and $53^{\circ}$ Earth incidence angles on the wind direction relative to the radiometer azimuthal look. For this, we have collocated brightness temperatures collected by the SSM/I and TMI microwave radiometers with wind vector measurements from buoys and the NASA scatterometer QUIKSCAT and independent satellite measurements of the columnar atmospheric water vapor and liquid cloud water. The wind direction signal is obtained from the difference between the measured brightness temperatures and the isotropic radiative transfer model function. It is important to use measurements of the atmospheric parameters that are uncorrelated with the brightness temperature measurements. This is necessary in order to avoid possible crosstalk between water vapor and liquid cloud water and relative wind direction, which prohibits the proper removal of atmospheric variations from the brightness temperatures.

We have analyzed three different data sets in the wind speed range between 0 and $14 \mathrm{~m} / \mathrm{s}$. The results for these three sets are very similar showing substantially smaller signals at low and intermediate wind speed than the earlier analysis by [18]. There is basically no signal below $5 \mathrm{~m} / \mathrm{s}$ for both $v$ and $h$-pol. At $8 \mathrm{~m} / \mathrm{s}$ their strengths are only about half of what had been previously reported. At higher wind speeds (above $12 \mathrm{~m} / \mathrm{s}$ ) we obtain the same results as [18]. We also find that the $v$-pol signal consists basically of a pure up-downwind asymmetry (1st harmonic), whereas the $h$-pol signal consists basically of a pure along-crosswind asymmetry (second harmonic) over the whole wind speed range. At lower frequencies, the strength of the emissivity signal drops approximately linearly with the logarithm of the frequency. Compared with the signal at $37 \mathrm{GHz}$, the signals at $19 \mathrm{GHz}$ and $11 \mathrm{GHz}$ are about $30 \%$ and $60 \%$ weaker, respectively. Our new results for the 19 and $37 \mathrm{GHz}$ channels are similar to the recent measurements by the JPL aircraft radiometer WINDRAD [22], [23]. The 2-scale surface emission model [31] predicts substantially larger dominant harmonics for low and intermediate wind speeds below $10 \mathrm{~m} / \mathrm{s}$ than we do. It agrees well with our new analysis and the JPL_-WINDRAD results at $10 \mathrm{~m} / \mathrm{s}$.

We have also studied the origin of the differences between our new analysis and [18]. We found that the small data set of [18] contained strong variations of the atmospheric parameters. It was not possible to remove these variations properly from the wind direction signal, because independent measurements of water vapor and liquid cloud water were not available in this case.

The new results reported here for $v$ and $h$-pol along with the new results for the 3rd and 4th Stokes parameters [22], [23] both indicate the wind direction signal for low to moderate winds is considerably smaller than previously reported in [18] and [20], respectively. The smaller wind direction signal will be of benefit for some applications and a clear drawback for others. For example when retrieving SST, the wind direction acts as an unwanted source of noise in the measured brightness temperatures producing a crosstalk error between SST and wind direction. The frequencies primarily used for retrieving SST are 7 and $11 \mathrm{GHz}$. Our analysis finds the signal at $11 \mathrm{GHz}$ to be $40 \%$ of its value at $37 \mathrm{GHz}$ and extrapolating from Fig. 3 we expect it at $7 \mathrm{GHz}$ to be only $20 \%$ of its value at $37 \mathrm{GHz}$. A small wind direction signal in these channels will benefit the SST retrievals. 
On the other hand, when considering the retrieval of wind direction using microwave radiometers, the smaller signal is clearly a disappointment. Below $5 \mathrm{~m} / \mathrm{s}$, which make for about $30 \%$ of all cases, there is virtually no signal for $v$-pol, $h$-pol, or the 4 th Stokes parameters and the third Stokes parameter is quite small (about $0.5 \mathrm{~K}$ peak-to-peak). We think it is unlikely that wind direction will be retrievable for winds below $5 \mathrm{~m} / \mathrm{s}$. The situation is more favorable for wind direction retrievals above $10 \mathrm{~m} / \mathrm{s}$, which covers about $20 \%$ of all ocean surface winds. Here all four Stokes parameters exhibit a strong directional dependence and it is likely that future satellite polarimetric radiometers will provide useful vector winds at these higher wind speeds. Between 5 and $10 \mathrm{~m} / \mathrm{s}$, which comprises about $50 \%$ of all cases, there is still considerable uncertainty about the vector wind retrieval performance.

\section{ACKNOWLEDGMENT}

The authors would like to thank A. Lipton, AER, for helpful remarks and comments during the preparation of the manuscript.

\section{REFERENCES}

[1] C. S. Cox, "Measurements of slopes of high frequency wind waves," $J$. Mar. Res., vol. 16, pp. 199-225, 1958.

[2] H. Mitsuyasu and T. Honda, "Wind-induced growth of water waves," $J$. Fluid Mech., vol. 123, pp. 425-442, 1982.

[3] P. M. Smith, "The emissivity of sea foam at 19 and $37 \mathrm{GHz}$," IEEE Trans. Geosci. Remote Sensing, vol. GE-26, pp. 541-547, Sept. 1988.

[4] W. C. Keller and J. W. Wright, "Microwave scattering and the straining of wind-generated waves," Radio Sci, vol. 10, pp. 139-147, 1975.

[5] M. L. Banner and I. R. Young, "Modeling spectral dissipation in the evolution of wind waves. Part I: Assessment of existing model performance," J. Phys. Oceanography, vol. 24, pp. 1550-1571, 1994.

[6] I. R. Young, L. A. Verhagen, and M. L. Banner, "A note on the bimodal directional spreading of fetch-limited wind waves," J. Geophys. Res. vol. 100, pp. 773-778, 1995.

[7] K. Ewans, "Observations of the directional spectrum of fetch-limited waves," J. Phys. Oceanography, vol. 28, pp. 495-512, 1998.

[8] P. A. Hwang, D. W. Wang, E. J. Walsh, W. B. Krabill, and R. N. Swift, "Airborne measurements of the wavenumber spectra of ocean surface waves. Part I: Spectral slope and dimensionless spectral coefficient," $J$. Phys. Oceanography, vol. 30, pp. 2753-2767, 2000.

[9] P. A. Hwang and D. W. Wang, "Directional distributions and mean square slopes in the equilibrium and saturation ranges of the wave spectrum," J. Phys. Oceanography, vol. 31, pp. 1346-1360, 2001.

[10] W. L. Jones, P. G. Black, D. M. Boggs, E. M. Bracalente, R. A. Brown, G. Dome, J. A. Ernst, I. M. Halberstam, J. E. Overland, S. Peteherych, W. J. Pierson, F. J. Wentz, P. M. Woiceshyn, and M. G. Wurtele, "Seasat scatterometer: Results of the Gulf of Alaska workshop," J. Sci., vol. 204, pp. 1413-1415, 1979.

[11] F. Wentz, A. Mattox, and S. Peteherych, "New algorithms for microwave measurements of ocean winds: Applications to seasat and the special sensor microwave imager," J. Geophys. Res., vol. 91, pp. 2289-2307, 1986.

[12] C.-Y. Chi and F. K. Li, "A comparative study of several wind estimation algorithms for spaceborne scatterometers," IEEE Trans. Geosci. Remote Sensing, vol. 26, pp. 115-121, Mar. 1988.

[13] F. M. Naderi, M. H. Freilich, and D. G. Long, "Spaceborn radar measurement of wind velocity over the ocean-An overview of the NSCAT scatterometer system," Proc. IEEE, vol. 79, pp. 850-866, June 1991.

[14] F. Wentz, "Specifying the NSCAT MLE Expected Variance Using the After-the-Fit Sigma0 Residual," Remote Sensing Systems, Santa Rosa, CA, Tech. Memo 121 097, 1997.

[15] A. Stoffelen and D. Anderson, "The ECMWF Contribution to the Characterization, Interpretation, Calibration and Validation of ERS-1 Scatterometer Backscatter Measurements and Their use in Numerical Weather Prediction Models," ECMWF, Reading, U.K., ESA Contract 9097/90/NL/BI, 1995
[16] A. Stoffelen, "Scatterometry," Ph.D. dissertation, Univ. Utrecht, Utrecht, The Netherlands, 1998.

[17] Y. A. Bespalova, V. M. Veselov, and V. Y. Gershenzon et al., "Determining surface wind velocity by measurements of polarization anisotropy of natural and scattered microwave radiation," Issledovaniye Zemli Iz Kosmosa, pp. 87-94, 1982.

[18] F. Wentz, "Measurement of oceanic wind vector using satellite microwave radiometers," IEEE Trans. Geosci. Remote Sensing, vol. 30, pp. 960-972, Sept. 1992.

[19] S. H. Yueh, W. J. Wilson, F. K. Li, S. V. Nghiem, and W. B. Ricketts, "Polarimetric measurements of sea surface brightness temperatures using an aircraft K-band radiometer," IEEE Trans. Geosci. Remote Sensing, vol. 33, pp. 85-92, Jan. 1995

[20] W. Wilson and S. Yueh, "Ocean Wind Direction Measurements Using Passive Polarimetric Radiometers," Tech. Rep., Jet Propulsion Lab., Pasadena, CA, JPL Publ. 96-6, 1996.

[21] S. H. Yueh, W. J. Wilson, F. K. Li, S. V. Nghiem, and W. B. Ricketts, "Polarimetric brightness temperatures of sea surfaces measured with aircraft $\mathrm{K}$ - and Ku-band radiometers," IEEE Trans. Geosci. Remote Sensing, vol. 35, pp. 1177-1187, Sept. 1997.

[22] S. Yueh, W. Wilson, S. Dinardo, and F. Li, "Polarimetric microwave brightness signatures of ocean wind directions," IEEE Trans. Geosci. Remote Sensing, vol. 37, pp. 949-959, Mar. 1999.

[23] S. Yueh and W. Wilson, "Validation of Wind Radiometer Technique Using Aircraft Radiometer and Radar Measurements for High Ocean Winds," Tech. Rep., Jet Propulsion Lab., Pasadena, CA, Publ. JPL D-17 815, 1999

[24] J. D. Jackson, Classical Electrodynamics. New York: Wiley, 1975.

[25] S. T. Wu and A. K. Fung, "A noncoherent model for microwave emission and backscattering from the sea surface," J. Geophys. Res., vol. 77, pp. 5917-5929, 1972

[26] F. Wentz, "A two-scale scattering model for foam-free sea microwave brightness temperatures," J. Geophys. Res., vol. 80, pp. 3441-3446, 1975

[27] H. A. Yueh, R. T. Shin, and J. A. Kong, "Scattering of electromagnetic waves form a periodic surface with random roughness," J. Appl. Phys., vol. 64, pp. 1657-1670, 1988.

[28] S. H. Yueh, R. Kwok, F. K. Li, S. V. Nghiem, W. J. Wilson, and J. A. Kong, "Polarimetric passive remote sensing of ocean wind vectors," Radio Sci., vol. 29, pp. 799-814, 1994.

[29] S. H. Yueh, "Modeling of wind direction signals in polarimetric sea surface brightness temperatures," IEEE Trans. Geosci. Remote Sensing, vol. 35, pp. 1400-1418, Nov. 1997.

[30] G. Poe and K. St. Germain, Polarimetric Emission Model of the Sea at Microwave Frequencies, Part I: Theory. Washington, DC: Naval Research Lab., 1998.

[31] K. St. Germain and G. Poe, Polarimetric Emission Model of the Sea at Microwave Frequencies, Part II: Comparison With Measurements. Washington, DC: Naval Research Lab., 1998.

[32] _ - "Modeling of polarimetric microwave signal due to ocean surface wind vector," in Proc. Int. Geosci. Remote Sensing Symp., Seattle, WA, 1998.

[33] - "Modeling of polarimetric microwave signal due to ocean surface wind vector," in Proc. 6th Spec. Meeting Microw. Radiometry Remote Sensing Environ., Florence, Italy, 1999.

[34] S. H. Yueh, R. Kwok, and S. V. Nghiem, "Polarimetric scattering and emission properties of targets with reflection symmetry," Radio Sci., vol. 29, pp. 1409-1420, 1994.

[35] H. J. Liebe, "An updated model for millimeter wave propagation in moist air," Radio Sci., vol. 20, pp. 1069-1089, 1985

[36] R. M. Goody and Y. L. Young, Atmospheric Radiation (Theoretical Basis), 2nd ed. New York: Oxford Univ. Press, 1989.

[37] F. T. Ulaby, R. K. Moore, and A. K. Fung, Microwave Remote Sensing: Active and Passive. Norwood, MA: Artech House, 1981, vol. 1.

[38] D. H. Staelin, K. F. Kunzi, R. L. Pettyjohn, R. K. L. Poon, R. W. Wilcox, and J. W. Waters, "Remote sensing of atmospheric water vapor and liquid water with the Nimbus-5 microwave spectrometer," J. Appl. Meteorol., vol. 15, pp. 1204-1214, 1976.

[39] P. W. Rosenkranz, "Shape of the $5 \mathrm{~mm}$ oxygen band in the atmosphere," IEEE Tran. Antennas Propagat., vol. AP-23, pp. 498-506, July 1975.

[40] P. Rosenkranz, "Water vapor microwave continuum absorption: A comparison of measurements and models," Radio Sci., vol. 33, pp. 919-928, 1998.

[41] H. J. Liebe and D. H. Layton, "Millimeter-Wave Properties of the Atmosphere: Laboratory Studies and Propagation Modeling," Tech. Rep., Natl. Telecommun. Inf. Admin., Boulder, CO, NTIA Rep. 87-224, 1987.

[42] H. J. Liebe, P. W. Rosenkranz, and G. A. Hufford, "Atmospheric 60-GHz oxygen spectrum: New laboratory measurements and line parameters," J. Quant. Spectrosc. Radiat. Transf., vol. 48, pp. 629-643, 1992. 
[43] H. J. Liebe, G. A. Hufford, and M. G. Cotton, "Propagation modeling of moist air and suspended water/ice particles at frequencies below 1000 GHz,” in Proc. AGARD'93 Conf., 1993.

[44] F. Wentz, "A model function for ocean microwave brightness temperatures," J. Geophys. Res., vol. 88, pp. 1892-1908, 1983.

[45] F. Wentz and T. Meissner, AMSR ocean algorithm (version 2), in Remote Sensing Systems, Santa Rosa, CA, Algorithm Theoretical Basis Document (ATBD), 1999.

[46] C. P. Yeang, S. H. Yueh, K.-H. Ding, and J. A. Kong, "Atmospheric effect on microwave polarimetric remote sensing of ocean surfaces," Radio Sci., vol. 34, pp. 521-537, 1999.

[47] F. J. Wentz, "A well calibrated ocean algorithm for special sensor microwave/imager," J. Geophys. Res., vol. 102, pp. 8703-8718, 1997.

[48] F. Wentz and R. Spencer, "SSM/I rain retrievals within a unified allweather ocean algorithm," J. Atmos. Sci., vol. 55, pp. 1613-1627, 1998.

[49] R. W. Reynolds, D. C. Stokes, and T. S. Smith, Global SST. Camp Springs, MD: National Center for Environmental Prediction, 1981.

Thomas Meissner (M'02) received the B.S. degree in physics from the University of Erlangen-Nürnberg, Germany, in 1983, the M.S. degree in physics from the University of Bonn, Germany, in 1987, and the Ph.D. degree in theoretical physics from the University of Bochum, Germany, in 1991. His doctoral dissertation was on effective quark models of the nucleon.

Between 1992 and 1998, he conducted postdoctoral research at the University of Washington, Seattle, the University of South Carolina, Columbia, and at Carnegie Mellon University, Pittsburgh, PA, in theoretical nuclear and particle physics focusing on the theory of strong interaction. Since July 1998, he has been with Remote Sensing Systems (RSS), Santa Rosa, CA. Since then, he has worked on calibration and validation for SSM/I and TMI, the development and refinement of radiative transfer models that relate satellite observations to geophysical parameters, and the development of a wind vector retrieval algorithm for CMIS.
Frank Wentz received the B.S. and M.S. degrees in physics from the Massachusetts Institute of Technology, Cambridge, in 1969 and 1971, respectively.

In 1974, he established Remote Sensing Systems, Santa Rosa, CA, a research company specializing in satellite microwave remote sensing of the earth. His research focuses on radiative transfer models that relate satellite observations to geophysical parameters, with the objective of providing reliable geophysical data sets to the earth science community. As a member of NASA's SeaSat Experiment Team (1978-1982), he pioneered the development of physically based retrieval methods for microwave scatterometers and radiometers. Starting in 1987 , he took the lead on providing the world-wide research community with high-quality ocean products derived from satellite microwave imagers (SSM/I). As the Director of RSS, he overseas the production and validation of reliable scatterometer and radiometer products. These data are dispersed via the company's web and ftp sites. He is currently working on scatterometer/radiometer combinations, satellite-derived decadal time series of atmospheric moisture and temperature, the measurement of sea-surface temperature through clouds, and advanced microwave sensor designs for climatological studies.

Mr. Wentz is a member of NASA Earth Observation System (EOS) Investigators Working Group, NASA Advanced Microwave Scanning Radiometer (AMSR) Team, NASA Ocean Vector Wind Science (OVWST) Team, NASA Tropical Rainfall Mission (TRMM) Team and NASA Pathfinder Activity. He has served on many NASA review panels, the National Research Council's Earth Studies Board, the National Research Council's Panel on Reconciling Temperature Observations, and is a member of the American Geophysical Union. 